\title{
OPEN Periplocin and cardiac glycosides suppress the unfolded protein response
}

\author{
Muneshige Tokugawa ${ }^{1}$, Yasumichi Inoue ${ }^{1,2 \varpi}$, Kan'ichiro Ishiuchi $^{3}$, Chisane Kujirai $^{1}$, \\ Michiyo Matsuno ${ }^{4}$, Masaki Ri ${ }^{5}$, Yuka Itoh ${ }^{1}$, Chiharu Miyajima ${ }^{1,2}$, Daisuke Morishita ${ }^{1,6}$, \\ Nobumichi Ohoka ${ }^{7}$, Shinsuke lida ${ }^{5}$, Hajime Mizukami ${ }^{4}$, Toshiaki Makino ${ }^{3}$ \& \\ Hidetoshi Hayashi ${ }^{1,2 \bowtie}$
}

The unfolded protein response (UPR) controls protein homeostasis through transcriptional and translational regulation. However, dysregulated UPR signaling has been associated with the pathogenesis of many human diseases. Therefore, the compounds modulating UPR may provide molecular insights for these pathologies in the context of UPR. Here, we screened small-molecule compounds that suppress UPR, using a library of Myanmar wild plant extracts. The screening system to track $X$-box binding protein 1 (XBP1) splicing activity revealed that the ethanol extract of the Periploca calophylla stem inhibited the inositol-requiring enzyme 1 (IRE1)-XBP1 pathway. We isolated and identified periplocin as a potent inhibitor of the IRE1-XBP1 axis. Periplocin also suppressed other UPR axes, protein kinase R-like endoplasmic reticulum kinase (PERK), and activating transcription factor 6 (ATF6). Examining the structure-activity relationship of periplocin revealed that cardiac glycosides also inhibited UPR. Moreover, periplocin suppressed the constitutive activation of XBP1 and exerted cytotoxic effects in the human multiple myeloma cell lines, AMO1 and RPMI8226. These results reveal a novel suppressive effect of periplocin or the other cardiac glycosides on UPR regulation, suggesting that these compounds will contribute to our understanding of the pathological or physiological importance of UPR.

The endoplasmic reticulum (ER) is the major organelle that mediates folding proteins, such as membrane or secretory proteins. Peptide chains newly synthesized from ribosomes are organized to their proper conformations in the ER. These peptides are then transferred to the Golgi apparatus for further post-translational modifications ${ }^{1}$. However, an aberrant subcellular milieu, including nutrient deprivation, hypoxia, the hypersecretion of proteins, or the repressed function of the ER, which causes ER stress, resulted in the excessive accumulation of misfolded proteins in the ER lumen ${ }^{2,3}$.

To cope with ER stress, a subcellular response called unfolded protein response (UPR) is induced ${ }^{4}$. UPR comprises three central ER-resident transmembrane proteins: protein kinase R-like endoplasmic reticulum kinase (PERK), inositol-requiring enzyme 1 (IRE1), and activating transcription factor 6 (ATF6), the activation of which ameliorates ER stress by temporarily decreasing 5 '-untranslated region (UTR)-dependent translation or up-regulating the transcription of genes in association with ER chaperones ${ }^{2,3}$. On the other hand, when excessive or prolonged ER stress is not relieved intracellularly, UPR removes the abnormal cells from healthy tissue by inducing programmed cell death ${ }^{5,6}$.

Aberrant UPR activation has been implicated in the pathology of various diseases, including diabetes, neurodegeneration, atherosclerosis, and cancer ${ }^{7}$. Thus, the compounds that modulate inevitable ER stress or

\footnotetext{
${ }^{1}$ Department of Cell Signaling, Graduate School of Pharmaceutical Sciences, Nagoya City University, Nagoya 467-8603, Japan. 'Department of Innovative Therapeutic Sciences, Cooperative Major in Nanopharmaceutical Sciences, Graduate School of Pharmaceutical Sciences, Nagoya City University, Nagoya 467-8603, Japan. ${ }^{3}$ Department of Pharmacognosy, Graduate School of Pharmaceutical Sciences, Nagoya City University, Nagoya 467-8603, Japan. ${ }^{4}$ The Kochi Prefectural Makino Botanical Garden, Kochi 781-8125, Japan. ${ }^{5}$ Department of Hematology and Oncology, Graduate School of Medical Sciences, Nagoya City University, Nagoya 467-8601, Japan. ${ }^{6}$ Chordia Therapeutics Inc., Kanagawa 251-0012, Japan. ${ }^{7}$ Division of Molecular Target and Gene Therapy Products, National Institute of Health Sciences, Kawasaki 210-9501, Japan. ${ }^{\circledR}$ email: yainoue@ phar.nagoya-cu.ac.jp; hhayashi@phar.nagoya-cu.ac.jp
} 
the pathological activation of UPR may provide the molecular basis for therapy for these diseases ${ }^{8}$. Therefore, we herein investigated small-molecule compounds that suppress UPR, using a library of Myanmar wild plant extracts.

The present results demonstrated that the ethanol extract of the Periploca calophylla stem (P. calophylla EtOH ex.) inhibited the activation of X-box binding protein 1 (XBP1) splicing in a screening assay using HEK293 cells that express the luciferase reporter gene fused with the XBP1 splicing region. Through activity-guided fractionation and subsequent structural analysis, periplocin ${ }^{9}$ was isolated as an active compound from the methanol extract of the P. calophylla stem. Periplocin suppressed IRE1-mediated XBP1 splicing, activation of the PERK-activating transcription factor 4 (ATF4) pathway, and ATF6 activation involving its fragmentation. In an analysis of the structure-activity relationship, various cardiac glycosides, including periplocin, suppressed $X B P 1$ splicing. Furthermore, periplocin suppressed the constitutive activation of XBP1 and exerted cytotoxic effects in the human multiple myeloma (MM) cell lines, AMO1 and RPMI8226. The results of the present study indicate the potential of periplocin and other cardiac glycosides as molecular candidates for novel inhibitors of the global UPR pathway.

\section{Results}

Ethanol extract of P. calophylla stem inhibited the activation of XBP1 splicing. To identify the small molecules that suppress UPR activation, we constructed a reporter system that enabled us to illustrate IRE1-mediated XBP1 splicing. IRE1 is activated by ER stress through autophosphorylation, which results in the splicing out of 26 specific nucleotides from XBP1 mRNA ${ }^{10}$. We established a cell line stably expressing the XBP1us-luc2 reporter plasmid in HEK293 cells (Fig. 1a). We used this system to screen a library of 700 Myanmar wild plant extracts to find extracts that inhibit the activation of XBP1 splicing. The part of results in the screening is shown in Supplementary Fig. S1. In the library, we found that the ethanol extract of $P$. calophylla stem suppressed XBP1 splicing induced by the ER stressors, tunicamycin (TM) and thapsigargin (TG) (Fig. 1b and Supplementary Fig. S2). The inhibitory effect on the intrinsic splicing of XBP1 mRNA was also observed by reverse transcription PCR (RT-PCR) (Fig. 1c).

To isolate active compounds from the P. calophylla stem, we obtained an extract of the P. calophylla stem using methanol, and subsequently performed activity-guided fractionation using the methanol extract. A scheme of the fractionation of the methanol extract is summarized in Supplementary Fig. S3. As a result of fractionation and purification, periplocin (molecular weight: 696) ${ }^{9}$ was characterized as an active compound in the extract of the P. calophylla stem (Fig. 2a).

Periplocin suppresses activation of the whole UPR pathway. We investigated whether periplocin suppresses IRE1-mediated XBP1 splicing. As shown in Fig. 2b, periplocin suppressed TM-induced XBP1 mRNA splicing. It is noted that the inhibitory effect on $X B P 1$ splicing of periplocin was stronger than that of $4 \mu 8 \mathrm{C}$, the inhibitor for IRE1-XBP1 axis ${ }^{11}$ (Fig. 2c). On the other hand, periplocin also inhibited the transcription of CCAAT/enhancer-binding protein homologous protein (CHOP), tribbles-related protein 3 (TRB3), and glucoserelated protein 78 (GRP78), the genes of which are induced by the activation of PERK or ATF6 (Fig. 2b). These results prompted us to speculate whether periplocin suppresses not only the IRE1 pathway, but also other UPR pathways including the PERK or ATF6 axis. Therefore, we examined the effects of periplocin on the PERK and ATF6 pathways. PERK is autophosphorylated under ER stress, followed by phosphorylation of eukaryotic initiation factor $2 \alpha(\mathrm{eIF} 2 \alpha)$, increasing selective translation that depend on mRNA containing 5 '-UTR, such as $\mathrm{ATF} 4^{12}$. The results demonstrated that periplocin suppressed the PERK phosphorylation with mobility shift and up-regulation of ATF4 expression (Fig. 2d). These suppressive effects were also observed in cells treated with selective inhibitor GSK2656157, which is well-known to prevent the PERK signaling ${ }^{13}$, indicating periplocin suppresses the PERK pathway. We also examined the effect of an integrated stress response inhibitor (ISRIB), which was characterized that blunts the integrated stress response (ISR) by antagonizing the inhibitory effect of phosphorylated eIF2 on eIF2B ${ }^{14}$. It should be noted that pretreatment of ISRIB suppressed ATF4 induction but not PERK phosphorylation under ER stress (Fig. 2d). Furthermore, to investigate whether periplocin prevents ATF6 pathway, we detected ATF6a protein fragmentation and ATF6-transcriptional activity using a reporter plasmid including the ER stress element (ERSE) ${ }^{15}$. As shown in Fig. 2e, the specific ATF6a inhibitor Ceapin$A 7^{16}$ decreased the ATF6a cleaved fragment (shown as CL, 50-75 kDa). Similarly, pretreatment with periplocin reduced the cleaved ATF6 $a$ bands (Fig. 2e). In addition, periplocin abrogated the TM-induced response of ERSE as well as Ceapin-A7 (Fig. 2f). These results indicate that periplocin suppressed both the PERK and ATF6 pathways, suggesting that it suppresses the activation of not only the IRE1 pathway, but also both the PERK and ATF6 pathways (Fig. 2g).

Cardiac glycosides and their aglycones suppress activation of the IRE1-XBP1 pathway. Periplocin, a member of cardenolides, was initially isolated from Cortex periplocae root bark ${ }^{17,18}$. Periplocin, like digoxin, digitoxin, and ouabain, belongs to a group with a chemical structure containing a steroid skeleton and carbohydrate moiety ${ }^{19}$. These compounds in the group are categorized as cardiac glycosides. Therefore, we assumed that other cardiac glycosides may also inhibit UPR activation. To elucidate this assumption, we evaluated the inhibitory activities on XBP1 splicing of cardiac glycosides or compounds containing steroid skeletons (these structures are shown in Supplementary Fig. S4). The results of the luciferase assay in XBP1us-luc2 stably expressing cells showed that digoxin, digitoxin, ouabain, and hellebrin suppressed XBP1 splicing at concentrations similar to periplocin. This suppressive effect was also observed with digitoxigenin, which is an aglycone of the digitoxin; however, its effects appeared to be weaker than that of the glycosides. On the other hand, other compounds with a steroid skeleton but not included in cardiotonic steroids, such as digitonin, stigmas- 
a

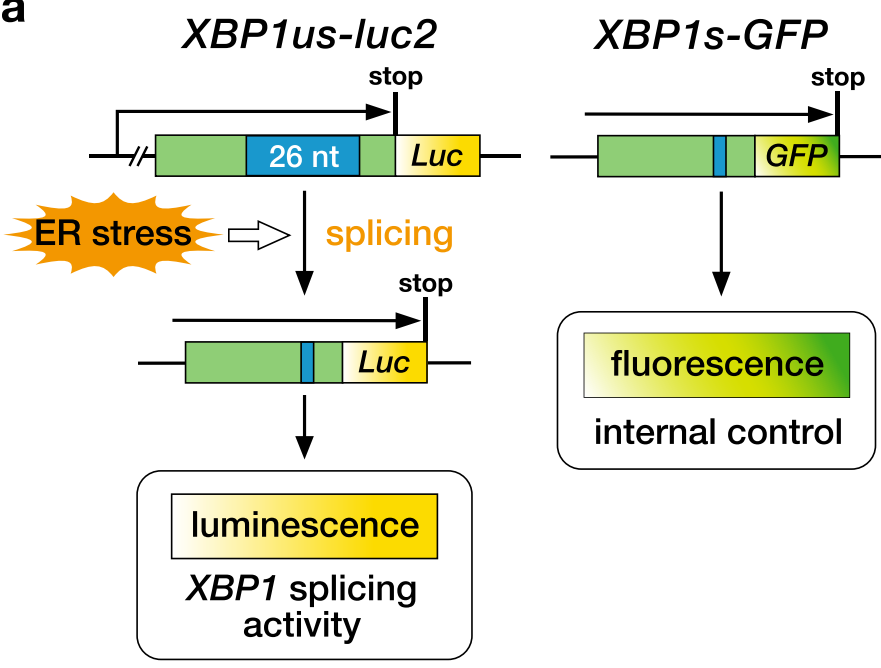

b

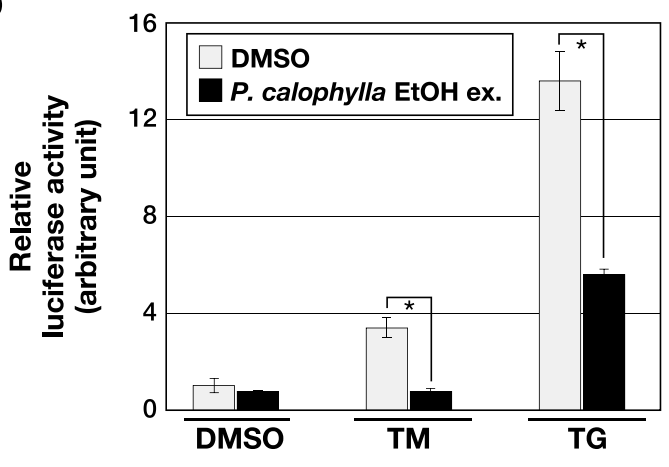

C

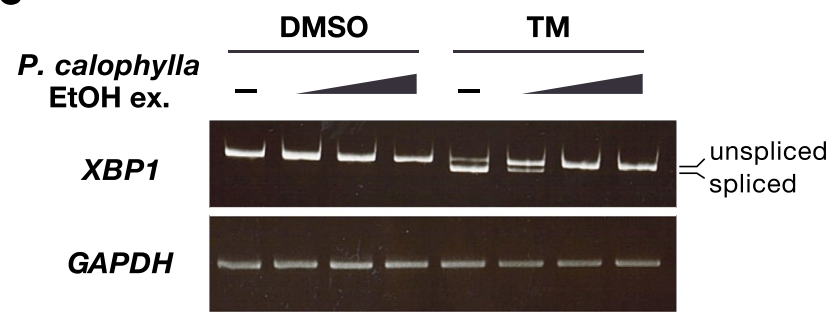

Figure 1. Ethanol extract of the P. calophylla stem inhibits activation of the IRE1-XBP1 pathway. (a) The scheme of the XBP1 splicing system (XBP1us-luc2 and XBP1s-GFP) in the reporter construct is shown. (b) HEK293 cells, stably expressing both XBP1us-luc2 and XBP1s-GFP, were pretreated with the ethanol extract of the $P$. calophylla stem (P. calophylla EtOH ex.) $(100 \mu \mathrm{g} / \mathrm{ml})$ for $1 \mathrm{~h}$, and were then incubated with tunicamycin (TM) $(0.5 \mu \mathrm{g} / \mathrm{ml})$ or thapsigargin (TG) $(0.5 \mu \mathrm{M})$. DMSO was added as vehicle. After $6 \mathrm{~h}$, luciferase activities and fluorescence intensities in cell lysates were measured. The luciferase activity was normalized by the fluorescence intensity. Values are shown as mean fold activity \pm standard deviations (S.D.) ( $n=3$ biological replicates). Statistical analysis was conducted between two groups with or without $P$. calophylla EtOH ex. for each TM or TG treatment assessed by two-tailed Student's $t$-test, and the significant differences are indicated as ${ }^{\star} p<0.05$. (c) HEK293 cells were pretreated with the P. calophylla EtOH ex. in a dose-dependent manner (10, 30, $100 \mu \mathrm{g} /$ $\mathrm{ml})$ for $1 \mathrm{~h}$, and were then incubated with TM $(0.5 \mu \mathrm{g} / \mathrm{ml})$ for $6 \mathrm{~h}$. The expression of each gene was assessed by RT-PCR. Unspliced or spliced XBP1 mRNA are indicated. GAPDH was used as the loading control. Uncropped images of gels are shown in Supplementary Information, Fig. S7.

terol, 18 $\beta$-glycyrrhetinic acid, hyodeoxycholic acid, and dexamethasone, did not suppress XBP1 splicing (Fig. 3). These results indicate that cardiac glycosides and their aglycones exhibit inhibitory activity against IRE1-mediated $X B P 1$ splicing.

Inhibitory effects of periplocin on constitutive activation of the IRE1-XBP1 pathway in multiple myeloma cells and its cytotoxic effect on cells. The IRE1-XBP1 pathway has been reported to be persistently activated and contribute to pathogenesis in some cancerous contexts. For example, survival or proliferation of multiple myeloma (MM) cells has been suggested to depend on the IRE1 axis. Indeed, the inhibition of XBP1 mRNA splicing was shown to induce apoptosis in MM cells ${ }^{20,21}$. The present results clearly demonstrated that periplocin inhibited the splicing of XBP1 mRNA caused by treatment with ER stressors (Figs. 1, 2 and 3). Moreover, periplocin has been reported to exhibit antitumor activity against several cancer cells ${ }^{19}$. These results led us to assume that periplocin showed suppressive effects on MM cell survival. To confirm the hypothesis, we investigated whether periplocin induced apoptosis in several MM cell line. In myeloma AMO1 and RPMI8226 cells, constitutive XBP1s expression was detected even under the absence of TM (Supplementary Fig. S5a). We also confirmed the position of XBP1s protein bands by HEK293 cells incubated with TM in the absence or presence of $4 \mu 8 \mathrm{C}$ (Supplementary Fig. S5b). Notably, the constitutive splicing of XBP1 mRNA and expression of XBP1s protein in AMO1 myeloma cells were inhibited in a dose-dependent manner following an exposure to periplocin (Fig. 4a). Periplocin also suppressed XBP1s protein levels with its time-dependent treatment in both AMO1 and RPMI8226 myeloma cells (Fig. 4b,c). Furthermore, dose- or time-dependent exposure of periplocin to these cells increased the level of truncated PARP and caspase 3, which are apoptosis markers 
a

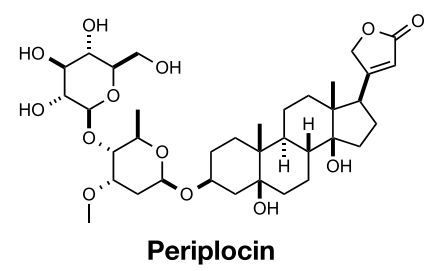

C

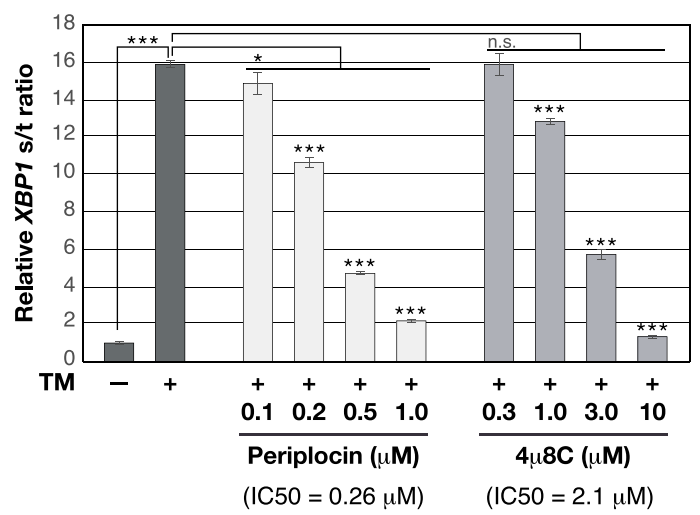

d

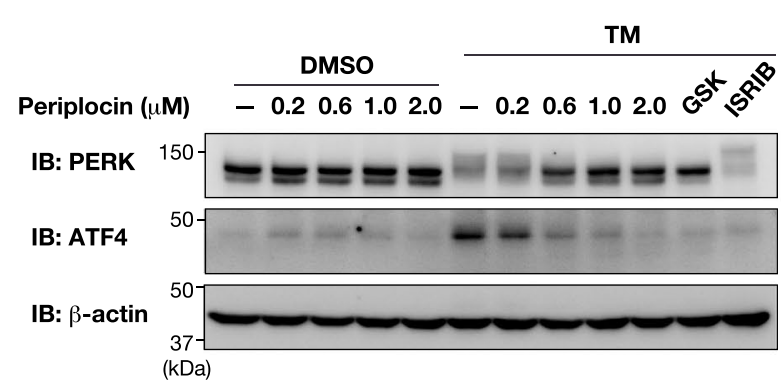

e

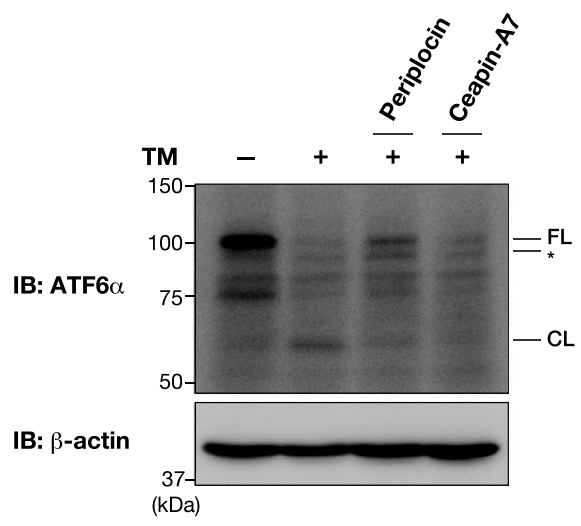

b

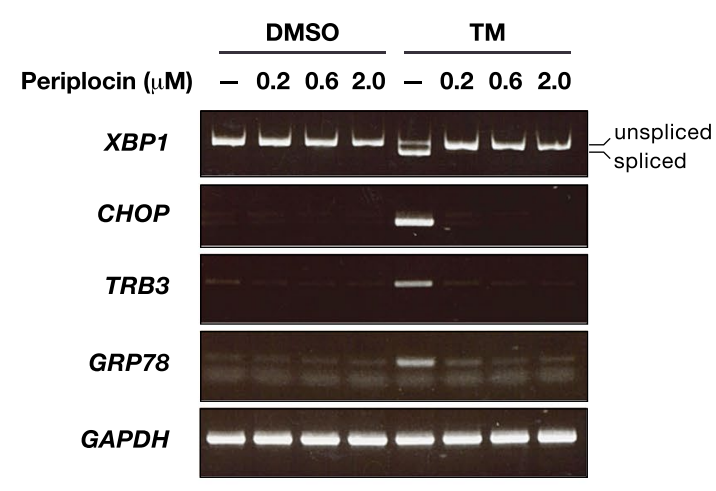

$\mathbf{f}$

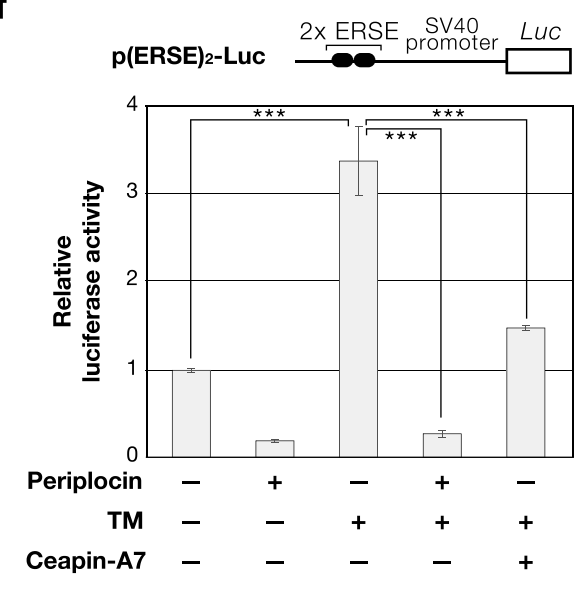

g

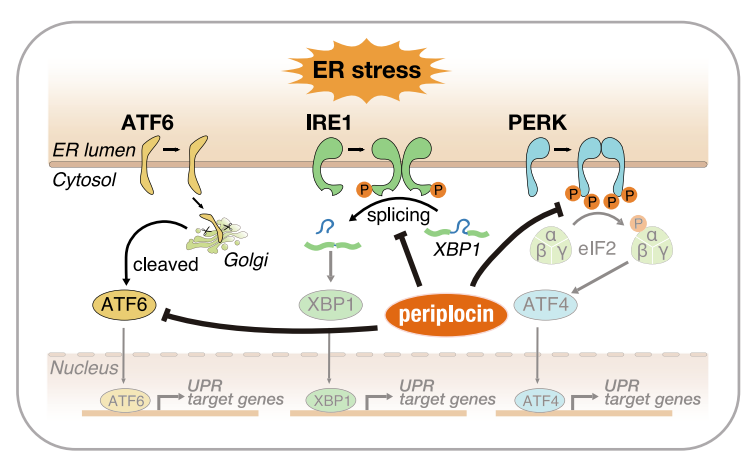


4Figure 2. Periplocin suppresses activation of the global UPR pathway. (a) Chemical structure of periplocin. (b) HEK293 cells were pretreated with the indicated doses of periplocin for $1 \mathrm{~h}$, and were then incubated with TM $(0.5 \mu \mathrm{g} / \mathrm{ml})$ for $6 \mathrm{~h}$. The expression of each gene was assessed by semi-qPCR. Unspliced or spliced XBP1 mRNA are indicated. GAPDH was used as the loading control. (c) HEK293 cells were pretreated with the indicated doses of periplocin for $1 \mathrm{~h}$, and were then incubated with TM $(0.5 \mu \mathrm{g} / \mathrm{ml})$ for $6 \mathrm{~h}$. Each expression of spliced $X B P 1 \mathrm{mRNA}$ and total XBP1 mRNA was quantified by real time qPCR. The ratio of spliced XBP1 mRNA/total $X B P 1$ mRNA $(X B P 1 \mathrm{~s} / \mathrm{t})$ are shown as mean fold changes \pm S.D. $(n=3$ technical replicates). IC50s for each of these compounds are shown. Significant differences are indicated as ${ }^{\star} p<0.05,{ }^{\star *} p<0.001$, assessed by one-way ANOVA with Dunnett's post-test; n. s., not significant. (d) HEK293 cells were pretreated with the indicated doses of periplocin, GSK2656157 (GSK, $1 \mu \mathrm{M})$ or ISRIB $(0.5 \mu \mathrm{M})$ for $1 \mathrm{~h}$, and were then incubated with TM $(0.5 \mu \mathrm{g} / \mathrm{ml})$ for $6 \mathrm{~h}$. Cell lysates were resolved by a $5-20 \%$ gradient gel and immunoblotted with the indicated antibodies. $\beta$-actin was used as the loading control. One representative from two independent experiments is shown. (e) HEK293 cells were pretreated with periplocin $(2 \mu \mathrm{M})$ or the ATF6a inhibitor Ceapin-A7 $(6 \mu \mathrm{M})$ for $1 \mathrm{~h}$, and were then incubated with TM $(2 \mu \mathrm{g} / \mathrm{ml})$ for $4 \mathrm{~h}$. The proteasome inhibitor MG132 $(10 \mu \mathrm{M})$ was added for $1 \mathrm{~h}$ prior to cell lysis to avoid degrading ATF6 $\alpha$ fragments. Full length of ATF6 $\alpha$ (FL), cleaved ATF6a (CL), and non-glycosylated ATF $6 a\left({ }^{*}\right)$ are indicated. $\beta$-actin was used as the loading control. One representative from two independent experiments is shown. (f) A schematic representation of the $\mathrm{p}(\mathrm{ERSE})_{2}$-Luc plasmid is shown (top). HEK293 cells were transiently transfected with $\mathrm{p}$ (ERSE) ${ }_{2}$-Luc and pCMV/ $\beta$-gal. After $24 \mathrm{~h}$, cells were pretreated with periplocin $(0.2 \mu \mathrm{M})$ or Ceapin-A7 $(6 \mu \mathrm{M})$ for $1 \mathrm{~h}$, and were then incubated with TM $(0.5 \mu \mathrm{g} /$ $\mathrm{ml})$ for $16 \mathrm{~h}$. Luciferase activity in cell lysates was measured and normalized by the $\beta$-galactosidase activity. Values are shown as mean fold activity \pm S.D. ( $n=3$ biological replicates). Significant differences are indicated as ${ }^{* * *} p<0.001$, assessed by one-way ANOVA with Dunnett's post-test. (g) A schema for the putative action of periplocin. Uncropped images of gels/blots are shown in Supplementary Information, Fig. S7.

(Fig. $4 \mathrm{a}-\mathrm{c}$ ). The viability of these cells was significantly reduced by the dose-dependent treatment with periplocin (Fig. 4d,e). Strikingly, the periplocin treatment increased the sub-G1 fraction including DNA-fragment of both AMO1 and RPMI8226 cells (Fig. 4f,g). These results suggest that periplocin has the potential to suppress $X B P 1$ splicing and reduce MM cell viability. In contrast, as shown in Supplementary Fig. S6, periplocin had little effect on the viability of normal human fibroblasts, TIG-1 cells. The constitutive splicing of XBP1 mRNA was not observed in TIG-1 cells (Supplementary Fig. S6). Collectively, the present results indicate that periplocin inhibits the ER stress-induced and constitutive activation of the IRE1-XBP1 pathway in MM cells. In addition, periplocin induced apoptosis in MM cells, but only had a negligible effect on normal cells.

\section{Discussion}

Recent findings indicated that excessive or chronical ER stress followed by UPR activation contributes to the pathogenesis of various diseases ${ }^{7}$. Thus, small molecules modulating UPR may help elucidate the relationship between UPR and diseases. Furthermore, approaches to control UPR activation are expected to contribute to the development of novel therapeutic interventions for UPR-related disorders, such as inflammatory diseases and cancer $^{8}$. Therefore, we herein attempted to identify novel active compounds that suppress UPR by monitoring the inhibitory effects on the IRE1-XBP1 pathway. We have succeeded in isolating periplocin from the methanol extract of $P$. calophylla as a potent inhibitor of the IRE1-XBP1 pathway.

We found that periplocin suppressed the activation of all three UPR branches in HEK293 cells (Fig. 2). For this reason, periplocin appears to affect the upstream pathway of UPR. In addition, the ethanol extract of $P$. calophylla or its active ingredient, periplocin, individually suppressed IRE1-mediated XBP1 splicing induced by different types of ER stressors TM or TG, respectively (Fig. 1,2). These results suggest that periplocin affects the factor(s) that modulates global UPR signaling, such as ER stress. Previous studies have reported that several compounds suppress UPR signaling ${ }^{11,16,22}$. 4-phenyl butyrate (4-PBA) or tauroursodeoxycholic acid (TUDCA), which is ER stress inhibitor, attenuates ER stress resulting in suppression of UPR activation ${ }^{23-25}$. Of note, unlike these compounds, periplocin is classically known to suppresses Na/K-ATPase activity as a cardiac glycoside ${ }^{19,26}$. However, pharmacological effect of suppressing Na/K-ATPase on UPR activation has been poorly elucidated. Interestingly, recent reports have shown that cardiac glycosides interact with $\mathrm{Na} / \mathrm{K}$-ATPase to modulate cellular signaling pathway, such as nuclear factor (NF)- $\kappa B$ axis or mitogen-activated protein kinase (MAPK) signaling ${ }^{26}$. Therefore, the suppressive effect of periplocin on UPR may be due to the regulation of Na/K-ATPase. To support this hypothesis, as shown in Fig. 3, only the cardiac glycosides periplocin, digoxin, digitoxin, ouabain, and hellebrin and an aglycone (digitoxigenin) suppressed IRE1-XBP1 activity. The suppressive effect of the aglycone on the IRE1-XBP1 axis was weaker than those of glycosides. Previous studies showed that the glycoside form of cardiac glycosides exhibited a stronger binding affinity to $\mathrm{Na} / \mathrm{K}$-ATPase than that of aglycones, which is more likely to influence inhibitory efficacy ${ }^{26,27}$. These findings indicate that the structural properties in cardiac glycosides required to depress $\mathrm{Na} / \mathrm{K}$-ATPase are key factors in inhibiting UPR. Although we did not clarify the protein(s) that periplocin targeted in UPR, the future identification of binding protein(s) for periplocin will provide insights into the novel molecular factor(s) regulating UPR.

In the UPR pathway, the IRE1-XBP1 axis has been shown to be associated with cancer progression and malignancy ${ }^{28,29}$. In drug-resistant malignant tumors, XBP1 mRNA is continuously spliced, and XBP1 modulates a number of genes associated with angiogenesis, cell proliferation, and metastasis ${ }^{30,31}$. Moreover, the IRE1 pathway has been implicated in MM. Munshi et al. showed that XBP1 mRNA was strongly expressed in patient-derived $\mathrm{MM}$ cells $^{32}$. Furthermore, transgenic mice overexpressing XBP1 exhibited pathological conditions similar to $\mathrm{MM}^{33}$. These findings indicate that the IRE1-XBP1 axis plays an essential role in the pathological mechanisms of 


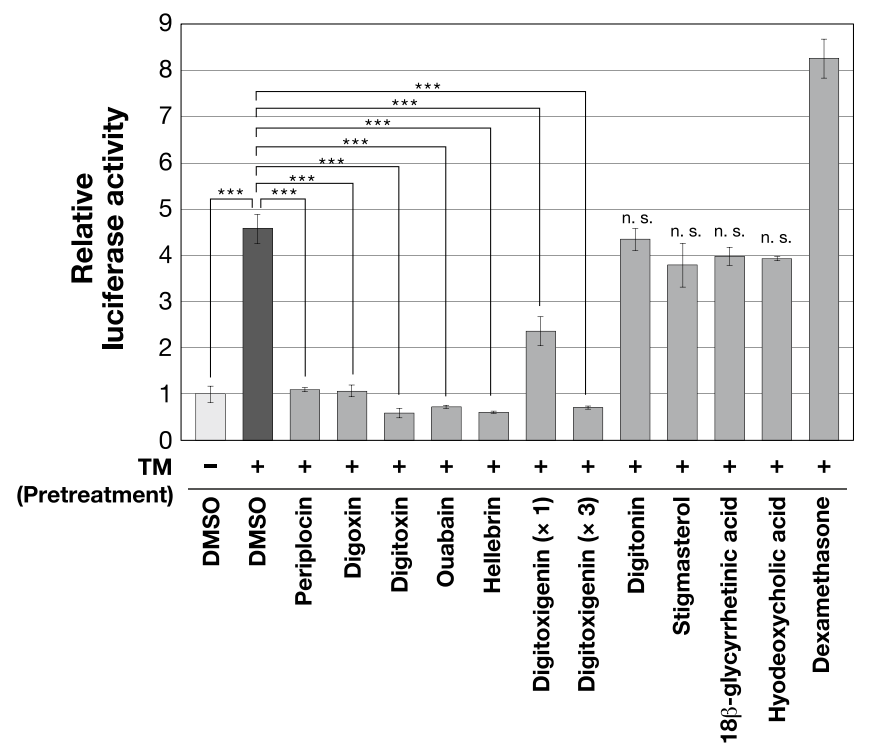

Figure 3. Various cardiac glycosides exert suppressive effects on UPR. HEK293 cells, stably expressing both $X B P 1 u s-l u c 2$ and $X B P 1 s-G F P$, were pretreated with cardiac glycosides $(0.2 \mu \mathrm{M})$ : periplocin, digoxin, digitoxin, ouabain, and hellebrin, or an aglycone $(\times 1: 0.2 \mu \mathrm{M}, \times 3: 0.6 \mu \mathrm{M})$ : digitoxigenin, or the other compounds that have a steroidal back bone, but do not exhibit cardiotonic activity $(0.2 \mu \mathrm{M})$ : digitonin, stigmasterol, $18 \beta$-glycyrrhetinic acid, hyodeoxycholic acid, and dexamethasone, for $1 \mathrm{~h}$ and were then incubated with TM $(0.5 \mu \mathrm{g} / \mathrm{ml})$. DMSO was added as vehicle. After $6 \mathrm{~h}$, luciferase activities and fluorescence intensities in cell lysates were measured. Luciferase activity was normalized by fluorescence intensity. Values shown are mean fold activity \pm S.D. ( $n=3$ biological replicates). Significant differences are indicated as ${ }^{\star * *} p<0.001$, assessed by oneway ANOVA with Dunnett's post-test; n. s., not significant.

several cancers including MM, and, hence, inhibitors for the IRE1-XBP1 pathway are currently being developed ${ }^{34}$. For example, toyocamycin and MKC-3946, which are small molecules that prevent IRE1 from splicing XBP1 mRNA, have the potential to depress cell proliferation and/or induce apoptosis in MM cells ${ }^{20,21}$. Similarly, our results using MM cells showed that periplocin inhibited constitutive XBP1 splicing, induced apoptotic cell death, and suppressed cell proliferation (Fig. 4). Previous studies on XBP1 silencing have reported marked reductions in the proliferation of MM cells ${ }^{21}$. These findings suggest that anticancer effects in MM cells exposed to periplocin were derived from the inhibition of the IRE1-XBP1 pathway.

In conclusion, periplocin suppressed the global UPR pathway by acting on unidentified factors. UPR signaling appears to be directly or indirectly associated with $\mathrm{Na} / \mathrm{K}$-ATPase, based on the structural properties or active moieties of periplocin and other cardiac glycosides. Periplocin also suppressed the survival of MM cells in which the IRE1-XBP1 pathway was constitutively activated. In addition to these suppressive effects, previous studies have indicated that periplocin has a variety of pharmacological properties, such as cardiotonic effects and anticarcinogenic and proapoptotic activities ${ }^{17,18}$. Several epidemiological studies have demonstrated that a treatment with cardiac glycosides prevented cancer recurrence and prolonged survival of cancer patients ${ }^{35}$. The suppression of the IRE1-XBP1 pathway by cardiac glycosides may effectively improve the prognosis of cancer patients, and this will be clarified in future studies. Further investigations will provide novel insights into the modes of action of periplocin by identifying its targeted protein(s), which may reveal mechanisms contributing to the physiological or pathological regulation of UPR.

\section{Methods}

Cell culture, plasmids, and establishment of a stable cell line. HEK293 and TIG1 cells were maintained in Dulbecco's modified Eagle's medium (DMEM) (Nacalai Tesque, Kyoto, Japan) supplemented with $10 \%$ fetal bovine serum (FBS) (Sigma, St. Louis, MO, USA), $100 \mathrm{U} / \mathrm{ml}$ of penicillin G, and $100 \mu \mathrm{g} / \mathrm{ml} \mathrm{of}$ streptomycin $^{36,37}$. AMO1 and RPMI8226 cells were cultured in Roswell Park Memorial Institute (RPMI) 1640 medium (Sigma) containing 10\% FBS and penicillin/streptomycin. Cells were grown in a $5 \% \mathrm{CO}_{2}$ atmosphere at $37^{\circ} \mathrm{C}$.

XBP1us-luc2 was constructed by ligating pcDNA3.1-Hygro (Invitrogen, Carlsbad, CA, USA) with an unspliced form of a Flag-XBP1 $\triangle \mathrm{DBD}$ fragment ${ }^{38}$, followed by the luc2 reporter gene derived from pGL4.10 (Promega, Madison, WI, USA). XBP1s-GFP was constructed by ligating pEGFP-N1 (TaKaRa Bio Inc., Shiga, Japan) with a spliced form of the Flag-XBP1 $1 \mathrm{DBD}$ fragment. $\mathrm{p}$ (ERSE) ${ }_{2}$-Luc was obtained from two tandem repeats of ERSE, which were amplified by PCR and ligated with the pGL3-Promoter Vector (Promega) ${ }^{39}$. pCMV/ $\beta$-gal was used as a plasmid expressing $\beta$-galactosidase ( $\beta$-gal).

HEK293 cells were transfected with XBP1us-luc2 using polyethylenimines (Polysciences, Warrington, PA, USA). After $48 \mathrm{~h}$, cells were selected with $100 \mu \mathrm{g} / \mathrm{ml}$ hygromycin. When colonies derived from a single cell were 
obtained, we subsequently transfected cells with XBP1s-GFP. Clonal cells expressing both XBP1us-luc2 and $X B P 1 s-G F P$ were used in experiments.

RNA extraction, semi-quantitative PCR (semi-qPCR), and real time qPCR (qPCR). Total RNA was extracted from cells as described previously ${ }^{40}$. First-strand cDNA was synthesized with the Prime Script first-strand cDNA Synthesis Kit (TaKaRa Bio Inc.) ${ }^{40}$. Semi-qPCR was performed as described previously ${ }^{41}$, using Gene Amp PCR System 2700 (Applied Biosystems, Foster City, CA, USA). Primers used for PCR were as follows: human CHOP, 5' -GCGTCTAGAATGGCAGCTGAGTCATTGCC-3' (forward) and 5' -GCGTCTAGATCA TGCTTGGTGCAGATTC-3' (reverse); human TRB3, 5' -TGCCCTACAGGCACTGAGTA-3' (forward) and 5' -GTCCGAGTGAAAAAGGCGTA-3' (reverse); human glyceraldehyde-3-phosphate dehydrogenase (GAPDH), 5' -TGAAGGTCGGAGTCAAAGGATTTGGT-3' (forward) and 5' -CATGTGGGCCATGAGGTCCACCAC-3' (reverse); human XBP1 (for Fig. 1c, 2b, Supplementary Fig. S6), 5' -GGAGTTAAGACAGCGCTTGG-3' (forward) and 5' -ACTGGGTCCAAGTTGTCCAG-3' (reverse); human XBP1 (for Fig. 4a), 5' -CGGAAGCCAAGG GGAATGAAG -3' (forward) and 5' -GGATATCAGACTCTGAATC-3' (reverse); human GRP78, 5' -TGAAGA GCTCAACATGGATCTGTT-3' (forward) and 5' -CTACAGCTTCATCTGGGTTTATGC-3' (reverse). A semiquantitative analysis for PCR products was performed using $8 \%$ polyacrylamide gel (for XBP1) or $1.5-2 \%$ agarose gel (for the others) electrophoresis. All the original data acquisition of gels is included in a Supplementary Fig. S7. qPCR was performed as described previously ${ }^{42}$. The specificities of the detected signals were confirmed by a dissociation curve, which consisted of a single peak. The primers used for qPCR were as follows: human $X B P 1 \mathrm{~s}$ (spliced), 5'-CTGAGTCCGCAGCAGGTG-3' (forward) and 5'-TCCAAGTTGTCCAGAATGCC-3' (reverse); human XBP1t (total), 5'-GGCATCCTGGCTTGCCTCCA-3' (forward) and 5'-GCCCCCTCAGCA GGTGTTCC-3' (reverse). The rate of spliced XBP1 mRNA/total XBP1 mRNA were calculated to show in Fig. 2c.

Immunochemical methods and antibodies. Immunoblotting was conducted as previously described ${ }^{43}$. In Fig. 2c, a gradient gel (SuperSep ${ }^{\mathrm{Tm}}$ Ace, 5-20\%; FUJIFILM Wako) was used for separating protein samples. The following commercially available antibodies were used: anti-PERK (\#5683; Cell Signaling Technology, Beverly, MA, USA), anti-ATF4 (\#11815; Cell Signaling Technology), anti-ATF6a (73-500; BioAcademia), anti-XBP1s (\#12782; Cell Signaling Technology), anti-PARP (\#9542; Cell Signaling Technology), anti-Cleaved Caspase-3 (Asp175) (\#9664; Cell Signaling Technology) and anti- $\beta$-actin (A5441; Sigma). All the original data acquisition of western blots is included in a Supplementary Fig. S7.

Reporter assay. HEK293 cells were transfected with the luciferase-expressing reporter, $\beta$-galactosidase, and empty vector. After $24 \mathrm{~h}$, cells were treated for the indicated periods with ER stressors. The luciferase assay was performed as previously described ${ }^{40}$. Values of luciferase activity were normalized to $\beta$-gal activity or the fluorescence intensity of GFP.

Cell viability assay and cell death assay. A cell proliferation assay was performed using water-soluble tetrazolium (WST)-8 (Dojindo, Kumamoto, Japan), as previously described ${ }^{44}$. AMO1 and RPMI8226 cells were plated at a concentration of $1 \times 10^{4}$ cells per well on a 96-well plate. Cells were then treated with periplocin for $24 \mathrm{~h}$. The WST- 8 reagent was added and cells were incubated at $37^{\circ} \mathrm{C}$ for $3 \mathrm{~h}$ in a $5 \% \mathrm{CO}_{2}$ atmosphere. Absorbance at $450 \mathrm{~nm}$ of the medium was measured. The percentage of cell proliferation was normalized to vehicletreated cells. Regarding the quantification of cell death, TIG1 cells were trypsinized and stained with trypan blue (Invitrogen) followed by counting with a hemocytometer. Cells stained blue were regarded as dead cells.

Sub-G1 fraction analysis. Cells were stained with propidium iodide using the Cycletest ${ }^{\mathrm{Tn}}$ Plus DNA Kit (BD Biosciences, Franklin Lakes, NJ, USA) according to the manufacturer's instructions. Changes in the DNA content in cells were detected using the FACSVerse ${ }^{\text {mat }}$ flow cytometer (BD Biosciences). The percentage of cells in the sub-G1 fraction was analyzed by FACSuite ${ }^{\text {Txx }}$ software (BD Biosciences).

Myanmar natural plant extract library and procedure of screening. The plant materials used to construct the 700 types of extract library, including the P. calophylla stem, were collected in Myanmar under the Memorandum of Understanding between the Kochi Prefectural Makino Botanical Garden (MBK), Japan and the Forestry Department (FD), the Ministry of Natural Resources and Environmental Conservation, Myanmar, in accordance with the Convention on Biological Diversity (CBD) and the Nagoya Protocol on access to genetic resources and benefit sharing. FD is the National Focal Point of Myanmar on CBD. Plant inventory in Myanmar has been performed under the permission from the Director General of FD. Voucher specimens of plant materials were deposited in the herbarium of MBK. Plant samples were equipped as an ethanol extract to the laboratory. Before use in screening, they were dissolved in DMSO to $100 \mathrm{mg} / \mathrm{ml}$. All extracts $(100 \mu \mathrm{g} / \mathrm{ml})$ were applied to cells expressing both XBP1us-luc2 and XBP1s-GFP for $1 \mathrm{~h}$ prior to incubation with tunicamycin $(0.5 \mu \mathrm{g} / \mathrm{ml})$ for $6 \mathrm{~h}$, and then the cell lysates were obtained. The XBP1 splicing activity was evaluated by the value of luciferase activity normalized by fluorescence intensity in the cell lysates. The extracts which decreased more than $50 \%$ of tunicamycin-induced $X B P 1$ splicing were determined as positive candidates in this screening.

Plant material. P. calophylla (Wight) Falc. was collected in the CHIN State in Myanmar. Botanical identification was performed by Dr. Kazumi Fujikawa, MBK. 
a
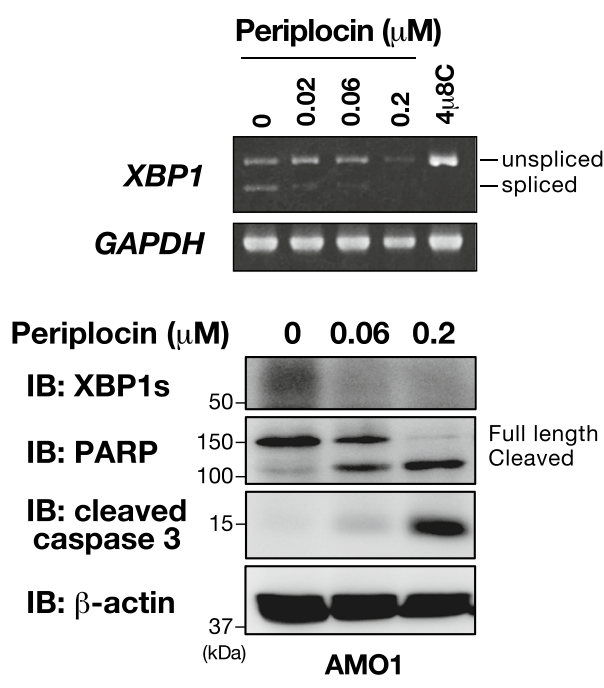

d

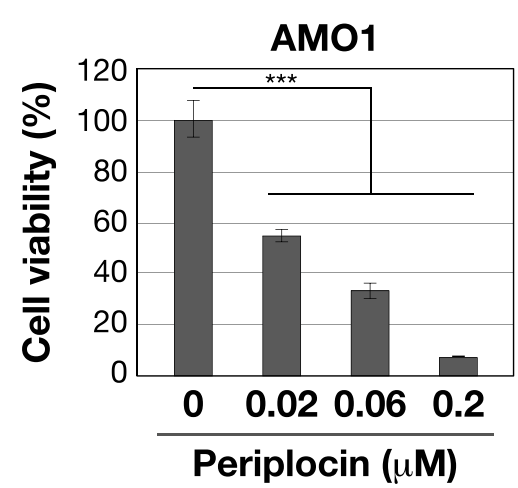

e

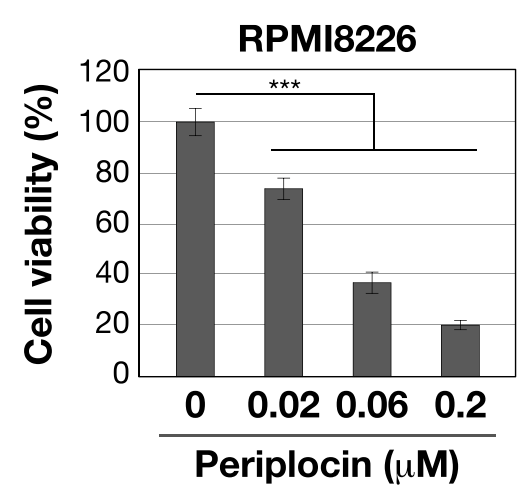

b

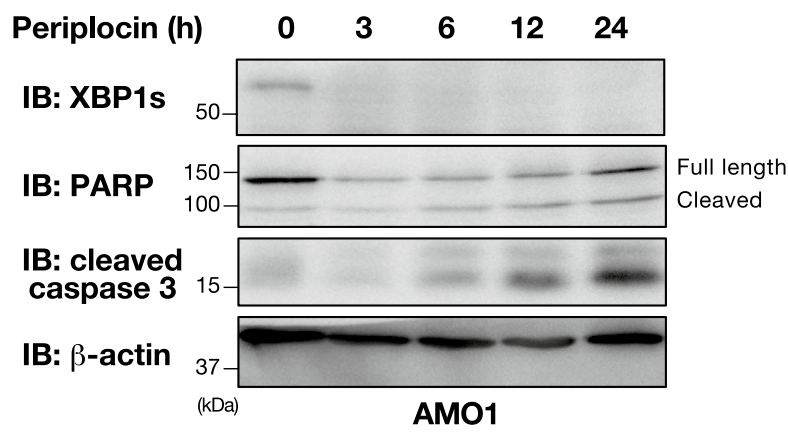

C

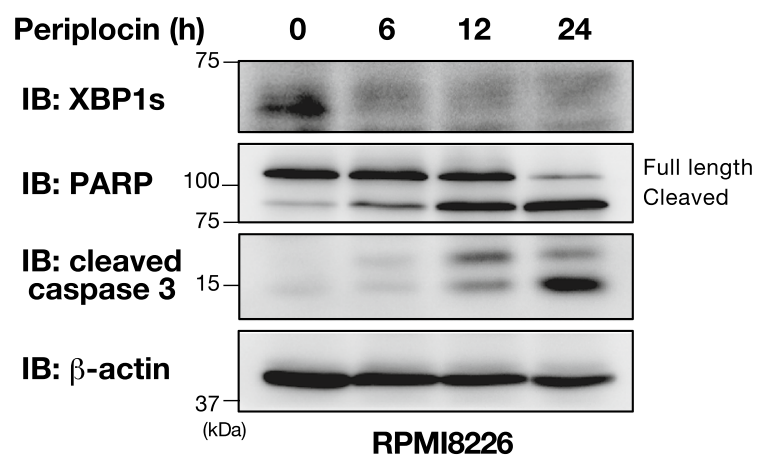

$\mathbf{f}$

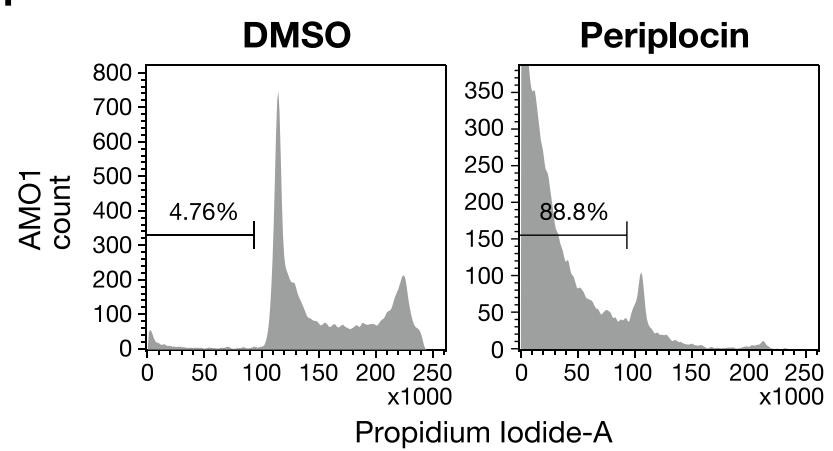

g

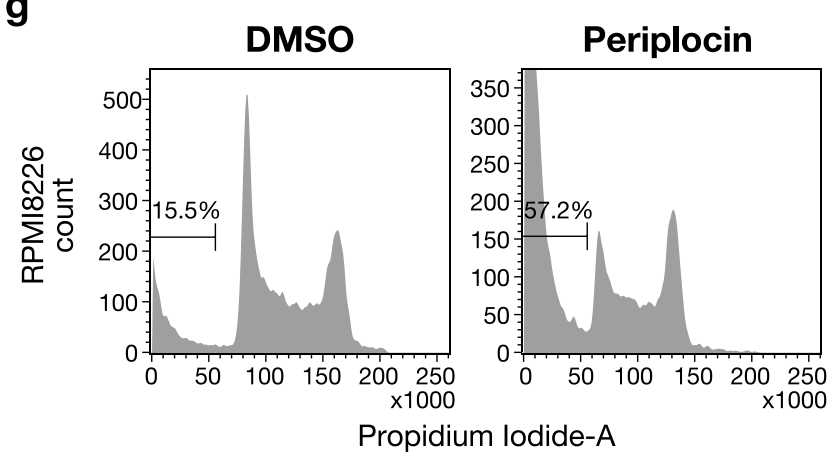


4Figure 4. Periplocin inhibits the constitutive activation of the IRE1-XBP1 pathway in multiple myeloma cells and exerts a cytotoxic effect on them. (a) For upper panel: AMO1 cells were incubated with the indicated doses of periplocin or $4 \mu 8 \mathrm{C}(10 \mu \mathrm{M})$ for $24 \mathrm{~h}$. The expression of each gene was assessed by RT-PCR. Unspliced or spliced XBP1 mRNA are indicated. GAPDH was used as the loading control. For lower panel: AMO1 cells were incubated with the indicated doses of periplocin for $24 \mathrm{~h}$. Cell lysates were immunoblotted with the indicated antibodies. $\beta$-actin was used as the loading control. In the immunoblotting one representative from two independent experiments is shown. (b, c) AMO1 (b) and RPMI8226 (c) cells were incubated with periplocin $(0.2 \mu \mathrm{M})$ for the indicated times. Cell lysates were immunoblotted with the indicated antibodies. $\beta$-actin was used as the loading control. One representative from two independent experiments is shown. (d, e) AMO1 (d) and RPMI8226 (e) cells were incubated with the indicated doses of periplocin for $24 \mathrm{~h}$. Cell viability was measured by the WST- 8 cell proliferation assay. Results are shown as mean \pm S.D. ( $n=3$ biological replicates). Significant differences are indicated as ${ }^{* *} p<0.001$, assessed by one-way ANOVA with Dunnett's post-test. $(\mathbf{f}, \mathbf{g})$ AMO1 (f) and RPMI8226 (g) cells were incubated with periplocin $(0.2 \mu \mathrm{M})$ or DMSO. After $24 \mathrm{~h}$, cells were stained with propidium iodide for a flow cytometric analysis of DNA content. The ratio of cells with DNA degradation is shown as a sub-G1 fraction and percentages are indicated in the histogram. Uncropped images of gels/blots are shown in Supplementary Information, Fig. S7.

Spectroscopic experimental procedures. Optical rotation was recorded on a JASCO P-2100 polarimeter. NMR spectra were recorded on an Agilent Varian VNS500 spectrometer. Chemical shifts (ppm) were referenced to residual solvent peaks $\left(\delta_{\mathrm{H}} 3.31\right.$ and $\delta_{\mathrm{C}} 49.0$ for $\left.\mathrm{CD}_{3} \mathrm{OD}\right)$. Positive-mode ESITOFMS was obtained on a JEOL JMS-T100LP AccuTOF LC-plus 4G spectrometer using a sample dissolved in $\mathrm{MeOH}$.

Extraction and isolation of periplocin from P. calophylla stem. The dried stem of P. calophylla (163 g) was extracted with methanol at room temperature and evaporated in vacuo. The concentrated methanol extract $\left(5.17 \mathrm{~g}\right.$ ) was successively partitioned among ethyl acetate, 1-butanol, and $\mathrm{H}_{2} \mathrm{O}$ to give active residues of the ethyl acetate and 1-butanol fractions, and inactive residues of the $\mathrm{H}_{2} \mathrm{O}$ fractions. The 1-butanol-soluble fraction was separated over a silica gel column using a stepwise gradient of increasing polarity from 100 to $0 \%$ chloroform in methanol into 11 sub-fractions. Two 1-butanol fractions (fraction 4, 5) were separated over an ODS-modified silica gel column using a stepwise gradient of decreasing polarity from 20 to $100 \%$ methanol aqueous solution into $10 \mathrm{sub}$-fractions. One of the $10 \mathrm{sub}$-fractions (fraction 7:25.7 $\mathrm{mg}$ ) was detected to have the potential to inhibit XBP1 splicing activity using a reporter assay. 1D and 2D NMR and ESI-MS analyses clarified that the active compound responsible for suppressing $X B P 1$ splicing was periplocin. Periplocin: $[\alpha]_{D}{ }^{27}+11(c$ 0.5, EtOH); ${ }^{1} \mathrm{H}-\mathrm{NMR}\left(\mathrm{CD}_{3} \mathrm{OD}, 500 \mathrm{MHz}\right) \delta 5.90(1 \mathrm{H}$, brs, H-22), $5.03(1 \mathrm{H}$, dd 18.5, $2.0 \mathrm{~Hz}, \mathrm{H}-21 \mathrm{a}), 4.91(1 \mathrm{H}$, dd 18.5, $2.0 \mathrm{~Hz}, \mathrm{H}-2 \mathrm{lb}), 4.86\left(1 \mathrm{H}, \mathrm{m}, \mathrm{H}-1^{\prime}\right), 4.34\left(1 \mathrm{H}, \mathrm{d} 7.5 \mathrm{~Hz}, \mathrm{H}-1^{\prime \prime}\right), 4.15(1 \mathrm{H}, \mathrm{m}, \mathrm{H}-3), 3.94$ (1H, ddd 3.0, 3.0, $3.0 \mathrm{~Hz}), 3.90\left(1 \mathrm{H}, \mathrm{m}, \mathrm{H}-6^{\prime \prime} \mathrm{a}\right), 3.88\left(1 \mathrm{H}, \mathrm{m}, \mathrm{H}-5^{\prime}\right), 3.64\left(1 \mathrm{H}, \mathrm{dd} 12.0,6.0 \mathrm{~Hz}, 6^{\prime \prime} \mathrm{b}\right), 3.46$ (3H, s, H-7'), 3.35 $\left(1 \mathrm{H}, \mathrm{m}, \mathrm{H}-4^{\prime}\right), 3.35\left(1 \mathrm{H}, \mathrm{m}, \mathrm{H}-3^{\prime \prime}\right), 3.27\left(1 \mathrm{H}, \mathrm{m}, \mathrm{H}-5^{\prime \prime}\right), 3.24\left(1 \mathrm{H}, \mathrm{m}, \mathrm{H}-4^{\prime \prime}\right), 3.20\left(1 \mathrm{H}, \mathrm{dd} 9.5,7.5 \mathrm{~Hz}, \mathrm{H}-2^{\prime \prime}\right), 2.84$ (1H, m, H-17), 2.18 (1H, m, H-4a), 2.16 (1H, m, H-16a), 2.14 (1H, m, H-15a), 2.10 (1H, brd 13.5 Hz, H-2'), $1.93(1 \mathrm{H}, \mathrm{m}, \mathrm{H}-7 \mathrm{a}), 1.88$ (1H, m, 16b), 1.78 (1H, m, H-2a), $1.72(1 \mathrm{H}, \mathrm{m}, \mathrm{H}-6 \mathrm{a}), 1.72$ (1H, m, H-15b), 1.71 $(1 \mathrm{H}, \mathrm{m}, \mathrm{H}-1 \mathrm{a}), 1.66(1 \mathrm{H}, \mathrm{m}, \mathrm{H}-2 \mathrm{~b}), 1.65(1 \mathrm{H}, \mathrm{m}, \mathrm{H}-8), 1.63(1 \mathrm{H}, \mathrm{m}, \mathrm{H}-2 \mathrm{~b}), 1.61(1 \mathrm{H}, \mathrm{m}, \mathrm{H}-9), 1.56(1 \mathrm{H}, \mathrm{m}$, H-4b), 1.50 (2H, H-12), 1.47 (1H, m, H-11a), 1.39 (1H, m, H-1b), 1.35 (1H, m, H-6b), 1.32 (1H, m, H-11b), $1.29\left(3 \mathrm{H}, \mathrm{d} 6.5 \mathrm{~Hz}, \mathrm{H}-6^{\prime}\right), 1.21(1 \mathrm{H}$, dddd 11.5, 11.5, 11.5, $4.0 \mathrm{~Hz}), 0.93(3 \mathrm{H}, \mathrm{s}, \mathrm{H}-19), 0.88(3 \mathrm{H}, \mathrm{s}, \mathrm{H}-18) ;{ }^{13} \mathrm{C}-$ NMR (CD $\mathrm{OD}, 125 \mathrm{MHz}) \delta 178.3(\mathrm{C}-20), 177.2$ (C-23), 117.8 (C-22), $106.2\left(\mathrm{C}-1^{\prime \prime}\right), 98.1\left(\mathrm{C}-1^{\prime}\right), 86.3(\mathrm{C}-14)$, $83.7\left(\mathrm{C}-4^{\prime}\right), 78.6\left(\mathrm{C}-3^{\prime}\right), 78.0\left(\mathrm{C}-5^{\prime \prime}\right), 77.9\left(\mathrm{C}-3^{\prime \prime}\right), 77.2(\mathrm{C}-3), 75.8(\mathrm{C}-5), 75.3(\mathrm{C}-21), 75.3\left(\mathrm{C}-2^{\prime \prime}\right), 71.8\left(\mathrm{C}-4^{\prime \prime}\right)$, 70.3 (C-5'), 63.0 (C-6"), 58.6 (C-7'), 51.9 (C-17), 50.9 (C-13), 41.8 (C-10), 41.7 (C-8), 40.9 (C-12), 40.2 (C-9), 36.6 (C-2'), 35.8 (C-4), 35.5 (C-6), 33.3 (C-15), 28.0 (C-16), 26.8 (C-2), 26.6 (C-1), 24.8 (C-7), 22.7 (C-11), 18.6 $\left(\mathrm{C}-6^{\prime}\right), 17.2$ (C-19), 16.3 (C-18); ESITOFMS m/z $719(\mathrm{M}+\mathrm{Na})^{+}$; HRESITOFMS m/z 719.3622 [M+Na $]^{+}(\mathrm{calcd}$ for $\left.\mathrm{C}_{36} \mathrm{H}_{56} \mathrm{O}_{13} \mathrm{Na}, 719.3619\right)$.

Chemicals. Periplocin (CAS: 13137-64-9) was commercially obtained from ChemFaces (Wuhan, China). Digoxin and digitoxin were purchased from Tokyo Kasei Kogyo (Tokyo, Japan). GSK2656157, ISRIB, digitoxigenin, and hellebrin were purchased from Cayman Chemical (Ann Arbor, MI, USA). The other chemicals were purchased from Sigma.

Statistical analysis. The significance of differences between two groups was assessed by the two-tailed Student's $t$-test. An analysis of significance for multiple groups was performed by a one-way ANOVA with the post hoc Dunnett's test.

Received: 5 February 2021; Accepted: 20 April 2021

Published online: 04 May 2021

\section{References}

1. Dobson, C. M. Protein folding and misfolding. Nature 426, 884-890 (2003).

2. Ron, D. \& Walter, P. Signal integration in the endoplasmic reticulum unfolded protein response. Nat. Rev. Mol. Cell Biol. 8, 519-529 (2007).

3. Hetz, C. The unfolded protein response: controlling cell fate decisions under ER stress and beyond. Nat. Rev. Mol. Cell Biol. 13, 89-102 (2012). 
4. Mori, K. Tripartite management of unfolded proteins in the endoplasmic reticulum. Cell 101, 451-454 (2000).

5. Puthalakath, H. et al. ER stress triggers apoptosis by activating BH3-only protein Bim. Cell 129, 1337-1349 (2007).

6. Han, J. et al. ER-stress-induced transcriptional regulation increases protein synthesis leading to cell death. Nat. Cell Biol. 15, 481-490 (2013).

7. Wang, M. \& Kaufman, R. J. Protein misfolding in the endoplasmic reticulum as a conduit to human disease. Nature 529, 326-335 (2016).

8. Hetz, C., Chevet, E. \& Harding, H. P. Targeting the unfolded protein response in disease. Nat. Rev. Drug Discov. 12, 703-719 (2013).

9. Sakuma, S., Ishizone, H., Kasai, R., Kawanishi, S. \& Shoji, J. Constituents of chinese crude drug "Wujiapi. " III. On the structure of glycoside G and K of Bei-Wujiapi. Chem. Pharm. Bull. 19, 52-59 (1971).

10. Lee, K. et al. IRE1-mediated unconventional mRNA splicing and S2P-mediated ATF6 cleavage merge to regulate XBP1 in signaling the unfolded protein response. Genes Dev. 16, 452-466 (2002).

11. Cross, B. C. et al. The molecular basis for selective inhibition of unconventional mRNA splicing by an IRE1-binding small molecule. Proc. Natl. Acad. Sci. U.S.A. 109, E869-E878 (2012).

12. Vattem, K. M. \& Wek, R. C. Reinitiation involving upstream ORFs regulates ATF4 mRNA translation in mammalian cells. Proc. Natl. Acad. Sci. U.S.A. 101, 11269-11274 (2004).

13. Atkins, C. et al. Characterization of a novel PERK kinase inhibitor with antitumor and antiangiogenic activity. Cancer Res. 73, 1993-2002 (2013).

14. Sidrauski, C., McGeachy, A. M., Ingolia, N. T. \& Walter, P. The small molecule ISRIB reverses the effects of eIF2a phopshorylation on translation and stress granule assembly. Elife 4, e05033. https://doi.org/10.7554/eLife.05033 (2015).

15. Yoshida, H. et al. Endoplasmic reticulum stress-induced formation of transcription factor complex ERSF including NF-Y (CBF) and activating transcription factors $6 \alpha$ and $6 \beta$ that activates the mammalian unfolded protein response. Mol. Cell. Biol. 21, 1239-1248 (2001)

16. Gallagher, C. M. \& Walter, P. Ceapins are new class of unfolded protein response inhibitors, selectively targeting the ATF6 a branch. Elife 5, e11878. https://doi.org/10.7554/eLife.11878 (2016).

17. Itokawa, H., Xu, J., Takeya, K., Watanabe, K. \& Shoji, J. Studies on chemical constitutes of antitumor fraction from Periploca sepium II Structures of new pregnane glicosides, periplocosides A, B and C. Chem. Pharm. Bull. 36, 982-987 (1988).

18. Kim, W. K. et al. Cytotoxic activities of Telectadium dongnaiense and its constituents by inhibition of the Wnt/ $\beta$-catenin signaling pathway. Phytomedicine 34, 136-142 (2017).

19. Li, Y. et al. A review on phytochemistry and pharmacology of Cortex periplocae. Molecules 21, 1702. https://doi.org/10.3390/molec ules21121702 (2016).

20. Ri, M. et al. Identification of Toyocamycin, an agent cytotoxic for multiple myeloma cells, as a potent inhibitor of ER stress-induced XBP1 mRNA splicing. Blood Cancer J. 2, e79. https://doi.org/10.1038/bcj.2012.26 (2012).

21. Mimura, N. et al. Blockade of XBP1 splicing by inhibition of IRE1 $\alpha$ is a promising therapeutic option in multiple myeloma. Blood 119, 5772-5781 (2012)

22. Axten, J. M. et al. Discovery of 7-Methyl-5-(1-\{[3-(trifluoromethyl)phenyl]acetyl\}-2,3-dihydro-1H-indol-5-yl)-7H-pyrrolo[2,3d]pyrimidin-4-amine (GSK2606414), a potent and selective first-in-class inhibitor of protein kinase R (PKR)-like endoplasmic reticulum kinase (PERK). J. Med. Chem. 55, 7193-7207 (2012).

23. Balch, W. E., Morimoto, R. I., Dillin, A. \& Kelly, J. W. Adapting proteostasis for disease intervention. Science 319, $916-919$ (2008).

24. Berger, E. \& Haller, D. Structure-function analysis of the tertiary bile acid TUDCA for the resolution of endoplasmic reticulum stress in intestinal epithelial cells. Biochem. Biophys. Res. Commun. 409, 610-615 (2011).

25. Basseri, S., Lhoták, S., Sharma, A. M. \& Austin, R. C. The chemical chaperone 4-phenylbutyrate inhibits adipogenesis by modulating the unfolded protein response. J. Lipid Res. 50, 2486-2501 (2009).

26. Mijatovic, T. et al. Cardiotonic steroids on the road to anti-cancer therapy. Biochim. Biophys. Acta. 1776, 32-57 (2007).

27. Katz, A. et al. Selectivity of digitalis glycosides for isoforms of human Na, K-ATPase. J. Biol. Chem. 285, 19582-19592 (2010).

28. Wang, M. \& Kaufman, R. J. The impact of the endoplasmic reticulum protein-folding environment on cancer development. Nat. Rev. Cancer 14, 581-597 (2014).

29. Cubillos-Ruiz, J. R., Bettigole, S. E. \& Glimcher, L. H. Tumorigenic and immunosuppressive effects of endoplasmic reticulum stress in cancer. Cell 168, 692-706 (2017).

30. Chen, X. et al. XBP1 promotes triple-negative breast cancer by controlling the HIF1a pathway. Nature 508, $103-107$ (2014).

31. Sheng, X. et al. IRE1a-XBP1s pathway promotes prostate cancer by activating c-MYC signaling. Nat. Commun. 10, 323. https:// doi.org/10.1038/s41467-018-08152-3 (2019).

32. Munshi, N. C. et al. Identification of genes modulated in multiple myeloma using genetically identical twin samples. Blood 103, 1799-1806 (2004).

33. Carrasco, D. R. et al. The differentiation and stress response factor XBP-1 drives multiple myeloma pathogenesis. Cancer Cell 11, 349-360 (2007).

34. Jiang, D., Niwa, M. \& Koong, A. C. Targeting the IRE1 $\alpha$-XBP1 branch of the unfolded protein response in human diseases. Semin. Cancer. Biol. 33, 48-56 (2015).

35. Menger, L. et al. Trail watch: cardiac glycosides and cancer therapy. Oncoimmunology 2, e23082. https://doi.org/10.4161/onci. $23082(2013)$.

36. Inoue, Y., Kitagawa, M. \& Taya, Y. Phosphorylation of pRB at Ser612 by Chk1/2 leads to a complex between pRB and E2F-1 after DNA damage. EMBO J. 26, 2083-2093 (2007).

37. Inoue, Y. et al. The CDK inhibitor p21 is a novel target gene of ATF4 and contributes to cell survival under ER stress. FEBS Lett. 591, 3682-3691 (2017).

38. Iwawaki, T., Akai, R. \& Kohno, K. A transgenic mouse model for monitoring endoplasmic reticulum stress. Nat. Med. 10, 98-102 (2004).

39. Ohoka, N., Yoshii, S., Hattori, T., Onozaki, K. \& Hayashi, H. TRB3, a novel ER stress-inducible gene, is induced via ATF4-CHOP pathway and is involved in cell death. EMBO J. 24, 1243-1255 (2005).

40. Inoue, Y., Abe, K., Onozaki, K. \& Hayashi, H. TGF- $\beta$ decreases the stability of IL-18-induced IFN- $\gamma$ mRNA through the expression of TGF- $\beta$-induced tristetraprolin in KG-1 cells. Biol. Pharm. Bull. 38, 536-544 (2015).

41. Kawarada, Y. et al. TGF- $\beta$ induces p53/Smads complex formation in the PAI-1 promoter to active transcription. Sci. Rep. 6, 35483. https://doi.org/10.1038/srep35483 (2016).

42. Fukuura, K. et al. The ubiquitin-specific protease USP17 prevents cellular senescence by stabilizing the methyltransferase SET8 and transcriptionally repressing $p 21$. J. Biol. Chem. 294, 16429-16439 (2019).

43. Inoue, Y. et al. Smad 3 is acetylated by p300/CBP to regulate its transactivation activity. Oncogene 26, 500-508 (2007).

44. Nagasaka, M. et al. Anti-tumorigenic activity of chrysin from Oroxylum indicum via non-genotoxic p53 activation through the ATM-Chk2 pathway. Molecules 23, 1394. https://doi.org/10.3390/molecules23061394 (2018).

\section{Acknowledgements}

The authors thank the members of the Hayashi laboratory for their helpful discussions. We also thank Dr. Shigeaki Hida (Nagoya City University) and Dr. Hisao Yamamura (Nagoya City University) for providing 
reagents and helpful discussions. We acknowledge the assistance of the Research Equipment Sharing Center at the Nagoya City University. This work was supported by a Grant-in-Aid for Scientific Research (C) (No. 18K06660, 20K07052) and a Grant-in-Aid for Scientific Research (B) (No. 21H02650) from the Japan Society for the Promotion of Science (JSPS), a Grant-in-Aid for Young Scientists (No. 18K16081) from JSPS, and a Grant-in-Aid for Research in Nagoya City University. This work was also supported by the Foundation of Oriental Medicine Study. MT was recipient of scholarship from Kidani Memorial Trust Foundation.

\section{Author contributions}

Ya.I., H.M., T.M., and H.H. conceived and designed the experiments. M.T., C.K., K.I., M.M., Yu.I., and C.M. performed the experiments. M.R., S.I., D.M. and N.O. contributed reagents and tools. M.T., Ya.I., and H.H. wrote the manuscript. All authors discussed the results and commented on the manuscript.

\section{Competing interests}

The authors declare no competing interests.

\section{Additional information}

Supplementary Information The online version contains supplementary material available at https://doi.org/ 10.1038/s41598-021-89074-X.

Correspondence and requests for materials should be addressed to Y.I. or H.H.

Reprints and permissions information is available at www.nature.com/reprints.

Publisher's note Springer Nature remains neutral with regard to jurisdictional claims in published maps and institutional affiliations.

(c) (i) Open Access This article is licensed under a Creative Commons Attribution 4.0 International License, which permits use, sharing, adaptation, distribution and reproduction in any medium or format, as long as you give appropriate credit to the original author(s) and the source, provide a link to the Creative Commons licence, and indicate if changes were made. The images or other third party material in this article are included in the article's Creative Commons licence, unless indicated otherwise in a credit line to the material. If material is not included in the article's Creative Commons licence and your intended use is not permitted by statutory regulation or exceeds the permitted use, you will need to obtain permission directly from the copyright holder. To view a copy of this licence, visit http://creativecommons.org/licenses/by/4.0/.

(C) The Author(s) 2021 